\title{
Role of sex and stature on the biomechanics of normal and loaded walking: implications for injury risk in the military
}

Niamh Gill ${ }^{{ }^{*}}$, Andrew J Roberts ${ }^{2}$, Thomas J O'Leary ${ }^{3,4}$, Anmin Liu ${ }^{1}$, Kristen Hollands ${ }^{1}$, Dale Walker ${ }^{1}$, Julie P Greeves ${ }^{3,4,5}$ and Richard K Jones ${ }^{1}$

${ }^{1}$ Centre for Health Sciences Research, University of Salford, Manchester, M6 6PU, UK.

${ }^{2}$ Army Recruit Health \& Performance Research, Army Recruit \& Initial Training Command, Upavon, SN9 6BE, UK.

${ }^{3}$ Army Health \& Performance Research, British Army, Andover, Hampshire, UK.

${ }^{4}$ Division of Surgery \& Interventional Science, University College London, London, UK

${ }^{5}$ Norwich Medical School, University of East Anglia, Norwich, UK.

* Corresponding author: N.M.Gill@salford.ac.uk (Niamh Gill)

\section{Keywords}

Marching; Load Carriage; Military; Injury; Walk to Run Transition; Biomechanics.

\section{Contributorship Statement}

Nimah Gill produced the manuscript. All authors edited the manuscript for intellectual content. All authors approved the final version of the manuscript.

\section{Funding}

This review was funded by the UK Ministry of Defence (Army) through the Analysis Support Construct (Task 0136).

\section{Competing interests}

The authors have no competing interests to declare.

\section{Acknowledgement}

None.

\begin{abstract}
Load carriage and marching 'in-step' are routine military activities associated with lower limb injury risk in service personnel. The fixed pace and stride length of marching typically vary from the preferred walking gait and may result in over-striding. Over-striding increases ground reaction forces and muscle forces. Women are more likely to over-stride than men due to their shorter stature. These biomechanical responses to over-striding may be most pronounced when marching close to the preferred walk-to-run transition speed. Load carriage also affects walking gait and increases ground reaction forces, joint moments and the demands on the muscles. Few studies have examined the effects of sex and stature on the biomechanics of marching and load carriage; this evidence is required to inform injury prevention strategies, particularly with the full integration of women in some defence forces. This narrative review explores the effects of sex and stature on the biomechanics of unloaded and loaded marching at a fixed pace and evaluates the implications for injury risk. The knowledge gaps in the
\end{abstract}


literature, and distinct lack of studies on women, are highlighted, and areas that need more research to support evidence-based injury prevention measures, especially for women in arduous military roles, are identified.

\section{Key Points}

- Marching 'in-step' at a fixed pace and stride length can result in over-striding in shorter individuals, increasing ground reaction and muscle forces, and injury risk.

- Marching at speeds close to the preferred walk-to-run transition speed may increase these forces and injury risk.

- Load carriage affects movement patterns and increases ground reaction forces, joint moments and the demands on the muscles.

- Few studies have examined the effects of sex and stature on the biomechanics of marching and load carriage.

- It is unknown whether men and women adapt their biomechanics differently when speed and stride length are enforced. 


\section{Introduction}

Military personnel are often required to carry heavy loads for prolonged periods. The load carried can exceed $70 \mathrm{~kg}$ in combat roles [1], depending on the operation, which is close to the body mass of some personnel. This heavy load carriage may increase injury risk [2-4], especially as the recommended maximum load that military personnel should carry is $45 \%$ of their body mass [5]. The incidence of musculoskeletal injuries ('injuries' herein) during basic training is high in the UK military (1.2 to $16.2 \%$ in men and 5.4 to $26.5 \%$ in women), and results in lost training days and medical discharge [6]. Women are 1.5 to 3.0 times more likely than men to sustain an injury during basic training [7,8], increasing their chance of re-injury or sustaining further injury [5]; women are also more susceptible to developing stress fractures, and hip injuries, than men [9]. The smaller stature and lower body mass of women likely contribute to their increased injury risk, particularly when carrying relatively heavier loads than men [10]. Currently, women make up around $10 \%$ of all service personnel in the UK Armed Forces [11], with a recruiting target of $15 \%$, and all roles are now open to them. Therefore, the biomechanical impact on women of operating in arduous roles needs to be fully understood to optimise training strategies and maximise through career opportunities.

Marching is a traditional military activity, performed in a regimented manner at a fixed pace and stride length. Walking with stride lengths longer than preferred increases the risk for lower limb and back injuries [12], due to increased joint loading [13, 14], while walking at speeds markedly different to the preferred walking speed results in an exponential increase in energy cost [15]. Furthermore, walking at speeds greater than the preferred walk-to-run transition speed is associated with greater rates of perceived effort [15]. Both preferred walking speed [16] and preferred walk-to-run transition speed $[17,18]$ are positively correlated with stature, in both men and women. However, the strength of the correlation between preferred walk-torun transition speed and stature varies considerably $(r=0.011$ to 0.690$)$, and only two studies include and present separate data on women. The effect of heavy load carriage on the preferred walk-to-run transition is not known [19].

Shorter stature, lower body mass and female sex are risk factors for lower limb injury in British Army recruits [6]. The independent effects of stature and sex on lower limb biomechanics of marching at a fixed stride length have not been examined. Moreover, the biomechanical effect of carrying relatively heavier loads ( $>30 \%$ of body mass), which better reflect the loads carried by military personnel wearing marching order $(59.4 \mathrm{~kg})$, is not fully understood. And, in some cases, military personnel may be required to carry heavier loads over shorter durations. A better understanding of biomechanical responses to changes in walking speed and stride length/frequency, and load carriage, will help inform preventative strategies for injury risk in military personnel. Most studies use male populations, and there is a need for more research to include women to determine if any sex-specific responses exist.

This narrative review aims to provide a broad summary of existing literature and present evidence on biomechanical risk factors for injury, focussing specifically on changes to walking gait and heavy load carriage, typical of military activities. 


\section{The role of fixed spatiotemporal parameters on biomechanics and the risk factors for injury during unloaded walking}

\section{Spatiotemporal parameters and walking speed}

Spatiotemporal parameters are characteristics of gait, which include stride length, stride frequency and speed. The preferred stride length for walking is, on average, $42 \pm 3 \%$ of an individual's stature $[20,21]$ and preferred walking speed is faster in those with taller stature and longer lower limb length [22]. Marching is performed at a fixed stride length and speed, irrespective of stature or limb length. This may force many individuals to walk with a greater stride length than preferred, increasing the risk of lower limb overuse injuries [23-25]. The shorter stature of women (mean (SD) stature for military personnel: men 1.77 (0.07) $\mathrm{m}$ and women $1.65(0.06) \mathrm{m}[10])$ reduces their preferred stride length and results in over-striding when marching in a mixed-sex group.

\section{Ground reaction forces}

Ground reaction forces (GRFs) are exerted on the body from contact with the ground. GRFs acting at a distance from a joint generates a turning effect, or torque, referred to as a joint moment. GRFs reportedly increase with increasing walking speed [26, 27], for example, Sun, Fekete [27] reported a 0.3 bodyweight increase in peak vertical GRF with a $25 \%$ increase in walking speed. Increased GRFs, and the corresponding increases in joint moments, are associated with overuse injuries, including stress fractures and knee joint problems [28]. Interestingly, Zadpoor and Nikooyan [29] showed that studies do not agree on whether there are significant differences in the GRFs and/or loading rates between stress fracture and control groups, during running. However, most studies included in this review compared groups running at a fixed speed and did not consider the effect of how increasing speed increases GRFs. The relationship between stride length and stride frequency on GRFs, normalised by body mass, are similar between sexes: as stride length increases, contact time, anterior-posterior braking and propulsive force, impulse descriptors, and vertical impulse per step systematically increase [30]. Interestingly, vertical peak forces and impulse per meter walked show little variation with changes in stride length [30]. However, it is worth noting that, relatively small ( \pm 5 and $\pm 10 \%$ ) changes in stride length and frequency were investigated, and therefore, the effect of greater changes in stride length on GRFs and lower limb biomechanics in men and women is unknown.

\section{Muscle forces}

Increasing stride length alone, to increase speed, results in a greater contribution to the vertical GRFs from the hip and knee muscles than increasing step frequency alone [31]. The (smaller) contribution from limb posture, defined as "the resistance to the downward pull of gravity provided by the bones and joints of the stance limb" [31, 32], increases as speed increases [31]; these mechanisms may explain the reduced walking speed and stride lengths that are observed in older adults with weak hip and knee extensors and may reflect an adaptation to alleviate the demand placed on the gluteus maximus and vasti muscles. This is significant when considering the injury risk of female soldiers marching in a group of men; as over-striding may exacerbate weakness in the muscles of the hip and knee that are required for counteracting the joint moments. Overall, lower limb muscle function was affected by changes in stride length more than step frequency [31]. However, participants in this study 
were required to walk at a "nominal" stride length $(0.73 \mathrm{~m})$ and step frequency $(1.92 \mathrm{steps} / \mathrm{s})$, and at $\pm 20 \%$ of these values. These "nominal" values were chosen to be representative of the normal for healthy young adults, based on published data, and would not account for differences in preferred stride length and step frequency. Therefore, participants may already have had to adjust their preferred walking biomechanics and so the effect of enforcing changes from preferred stride lengths and frequencies on lower limb muscle forces remains unknown.

\section{Preferred walking speed and preferred walk-to-run transition speed}

Humans have a preferred walking speed of approximately $1.4 \mathrm{~m} \cdot \mathrm{s}^{-1}$, on average [33]. Humans adopt a combination of preferred stride length and stride frequency to achieve their preferred walking speed. Walking at speeds markedly different to the preferred walking speed is associated with an exponential increase in energy cost [15] as well as increases in joint moments, particularly at the hip [13,31,34], which may increase the risk of lower limb and lower back injuries. Furthermore, as speed increases, there is a spontaneous transition between walking and running, known as the preferred walk-to-run transition speed [22]. The preferred walk-to-run transition speed is around $2 \mathrm{~m} \cdot \mathrm{s}^{-1}$ for healthy adults [18, 22]. However, this preferred speed is dependent on multiple factors (e.g. stature, limb length, and metabolic and mechanical efficiency) $[13,15,17,18,22,35]$, and is said to decreases when carrying external load [36], and occurs at a slower speed in women than men [18, 37].

The strength and significance of associations are mixed between the preferred walk-to-run transition speed and anthropometry (stature, sitting height, leg length, lower leg length, thigh girth and calf girth) $[17,18,36,38]$. Greater variability in stride length, frequency and duration is also observed at speeds near the preferred walk-to-run transition speed, suggesting a loss of stability in the movement pattern [39], increased rate of perceived effort [15, 40] and increased muscle activity $[40,41]$. These findings are relevant and important to a military population marching 'in-step'. Firstly, Army personnel must complete a $12.8 \mathrm{~km}$ load carriage at $\sim 1.8 \mathrm{~m} \cdot \mathrm{s}^{-1}$ [42] as part of their annual physical employment test, which is close to the average preferred walk-to-run transition speed for healthy adults [18, 22]. Secondly, a mixed military cohort will have a wide range of statures (mean (SD) statures for military personnel: men 1.77 (0.07) $\mathrm{m}$ and women 1.65 (0.06) m [10]), and, consequently, preferred walking speeds, walk-to-run transition speeds and stride lengths. To mitigate the biomechanical stress of over-striding, shorter personnel are typically placed at the front of a group to 'control' speed (when unspecified) and stride length. This formation will not fully prevent some individuals from marching at speeds closer to their preferred walk-to-run transition speed than their preferred walking speed, which likely results in less efficient marching [15], and increased joint loading [13, 31, 34].

Many studies have investigated the relationship between spatiotemporal parameters and walking biomechanics, and over-striding has been shown to increase injury risk [23, 24, 43]. However, the effects of sex and stature on the biomechanical response to enforcing spatiotemporal parameters are unknown. Moreover, whether the effect of stature on the preferred walk-to-run transition speed is sex dependent is also unclear. 


\section{The effect of load carriage on spatiotemporal parameters, biomechanics and injury risk}

A better understanding of the additive effect of load carriage on the biomechanical response to fixed walking speed, stride frequency and stride length will help mitigate injury risk in military personnel. A high number of studies have investigated the effect of load carriage on biomechanical function and metabolic energy expenditure in both civilian [21, 44-51] and military [34, 44, 49, 52-54] populations. However, only a proportion of these studies included women, and only four compared between sexes. This section will describe the effect of load carriage on walking biomechanics and, where data are available, differences between men and women.

\section{Spatiotemporal parameters and walking speed}

Studies investigating the effect of load carriage on stride length and frequency report mixed findings. Several studies have reported decreased stride length [44-47, 49-52, 54] and increased stride frequency $[47,50,52,54]$ with the increased load carried at self-selected and fixed $(1.5 \mathrm{~m} / \mathrm{s})$ walking speeds; where the loads carried ranged from approximately 0-65 \%body mass. Others reported no effect of the increased load carried on stride length [21, 48] or stride frequency $[44,51,52]$. One study reported decreased walking speed, stride length and stride frequency with increasing load (0-60 \%body mass) [47]. In contrast, another reported increased stride length and stride frequency with increased load [53], however, these increases were likely a result of the corresponding increase in walking speed and the relatively small loads considered $(0-17 \mathrm{~kg})$.

Similarly, there is conflicting evidence for sex differences in spatiotemporal measures during load carriage [21, 49]. In two studies, no sex differences were shown in GRFs, stride length or stride frequency while walking with loads at either the preferred walking speed [21] or a prescribed speed of $1.50 \mathrm{~m} \cdot \mathrm{s}^{-1}$ [49]. In contrast, women walked with higher stride frequencies than men at set speeds of $1.36 \mathrm{~m} \cdot \mathrm{s}^{-1}[55]$ and $1.78 \mathrm{~m} \cdot \mathrm{s}^{-1}$ [56]. Also, as load increased, women had a greater increase in stride frequency than men [56]. At a fixed speed of $1.36 \mathrm{~m} \cdot \mathrm{s}^{-1}$, stride length increased over time, and decreased with heavier loads, in women, but not in men; although, the findings may have been biased by the high number of women who may have had naturally shorter stride lengths [55] and were unable to complete the task.

These different spatiotemporal responses to load may, in part, be due to variability in participant populations (particularly load carriage experience) and the variability in loads carried, and/or reflect a learned effect or a non-linear response to load. Load carriage experience affects biomechanical adaptations, and military personnel who are familiar with marching 'in-step' are accustomed to maintaining stride length, even during load carriage [50]. However, over-striding is considered a risk factor for overuse injuries [23, 24, 43] and, therefore, shortening stride length is proposedly a mechanism for reducing biomechanical stress while carrying load [2]. The additional load may also reduce the mechanical efficiency of walking and decreases preferred walk-to-run transition speeds [36]. The effect of modifying spatiotemporal parameters during load carriage on lower limb biomechanics in men and women may help inform injury prevention measures.

\section{Kinematics}

Joint kinematics is the motion between two consecutive segments of the human body. Measurement of joint kinematics may improve our understanding of injury mechanisms, and 
the changes observed in energy expenditure, with load carriage [3, 12, 25, 57]. Changes in movement patterns are a likely mechanism to maintain postural control and alignment of the applied GRFs, as well as minimise the energetic cost of load carriage. The postural changes during load carriage may result in measurable changes in kinematic parameters [53], however, study findings are conflicting.

A number of other studies [44, 52, 58-60], and a recent meta-analysis [57], identified an association between load carriage and increased hip sagittal range of motion (RoM) [52, 57], unchanged knee sagittal RoM [57, 58], increased ankle sagittal RoM [45, 53, 57] and unchanged trunk sagittal RoM [52, 57]. However, others have reported decreased knee sagittal RoM [44, 59, 60], increased knee sagittal RoM [2, 52] and increased knee flexion [21, $53,61]$ with increased load carriage $(0-50 \mathrm{~kg})$. Differences in findings between studies may be explained by variations in load carriage experience, walking speed, hip belt usage and posterior displacement of the load away from the trunk. The biomechanical changes induced by load carriage are also influenced by the phase of walking $[45,53,57]$.

Inconsistent findings have also been reported for ankle kinematics. Some studies reported increased ankle dorsiflexion with increasing load $[52,53,59]$, however, others observed no change [34, 62]. Furthermore, adding load decreased ankle RoM when barefoot, but increased ankle RoM when shod during treadmill walking [45]. However, these findings were not replicated in a subsequent study investigating overground walking [46]. These conflicting results could be due to different footwear, or walking modalities, influencing ankle kinematics and/or by measurement error with identifying the underlying bony landmarks when wearing footwear.

Increased forward lean of the trunk with increasing (posterior) load is well documented [34, $52,59,60]$. Forward lean keeps the load centre of mass as close to the base of support as possible. Increased forward lean leads to flexion at the hip [21, 34, 52, 53, 59, 60, 62] that is counteracted by increased muscle activity in pelvic and low back musculature [34, 59, 60]. Load carriage may, therefore, increase the risk of lower back injuries due to the increased stress placed on the local musculature [52].

Krupenevich, Rider [49] observed significant sex by load interaction for average trunk position during walking. Women increased trunk lean to a greater extent than men during load carriage (13\% and $11 \%$, respectively); possibly due to their lighter mass and the need to lean further forward to offset the same load. Furthermore, a significant correlation was found between trunk lean and body mass, for the combined dataset of men and women, indicating lighter participants carried the load with greater forward lean [49]. Some epidemiological studies support the role of smaller body size, such as smaller body mass, shorter stature and shorter preferred stride length, rather than female sex, as principal risk factors for injury in basic military training [63-65]. Therefore, discerning the independent effects of sex and physical characteristics on biomechanical adaptations to load carriage is important for targeted injury mitigation strategies.

\section{Ground reaction forces and kinetics}

Overuse injuries result from repeated loading of the skeleton and soft tissue during training and operational tasks, with inadequate recovery [66]. During basic military training, recruits generally experience increased unaccustomed stresses, which may be relatively higher in 
women [67], including increased joint loading from running and load carriage [66]. Measurement of joint kinetics — the forces/moments that cause movement at the joint — and GRFs will improve our understanding of the biomechanical risk factors of injury with load carriage.

Load carriage increases the first and second peaks, and the minima between peaks, in the vertical GRF; the braking and propulsive GRF peak; and, the vertical, braking and propulsive impulse [57, 59, 60, 68]. Vertical GRFs increase proportionally with increasing load [2, 21, 46, $48,69]$. Silder, Delp [21] reported increased vertical GRF by approximately $6 \%$ for each $10 \%$ increase in load; no sex differences were detected for peak vertical GRFs normalised to body mass, although women were typically $12 \mathrm{~kg}$ lighter suggesting a lower absolute peak vertical GRF. A similar proportional increase is observed between the braking and propulsive GRFs and increasing load [2, 46, 69].

Peak hip and knee, flexor and extensor moments, and peak ankle plantar flexor moments increase with increasing load [21, 46, 61, 70, 71]. Although the relative increase in joint loading due to load carriage is likely speed dependent, the largest increase in joint moment consistently occurs at the knee joint [46, 70]. This increased knee joint moment may be a mechanism for dissipating energy and reducing joint loading elsewhere [70], however, this has not been experimentally tested. Furthermore, as load carriage will increase GRFs, joint moments will also likely increase and the muscles crossing joints will be required to produce more force to counteract these, which may lead to a greater risk of injury. The lack of sex differences suggests that the forces generated from load carriage are not attributable to the increased injury risk in women [21]; however, the intrinsic ability to cope with these loads could be a differentiating factor.

Changes to stride length and stride frequency affect the GRFs during loaded walking [12, 72]. At a fixed speed, when stride frequency decreases (and stride length increases) the anteriorposterior GRF increases [12]. Whereas at self-selected speeds, all GRF peaks were higher at a high stride frequency $(1 \mathrm{~Hz})$ compared with a low stride frequency $(0.6 \mathrm{~Hz})$ [72]. Importantly, as stride frequency decreased the knee extensor moment increased, which could have implications for the development of muscle fatigue; however when stride frequency increased there was no change to the knee extensor moment [12]. These findings are important when considering marching 'in-step,' where shorter individuals, who are more likely to be women, must over-stride to match stride lengths of taller individuals; shorter individuals will experience greater stress on the knee extensor muscles compared with marching at their preferred stride length/frequency [12].

Despite the high number of studies investigating the biomechanics of load carriage, in both military and civilian populations, there are still gaps in our understanding. Specifically, there are very few studies comparing the effects of load carriage between the biomechanics of men and women. Women are typically smaller and have less muscle mass than men [67, 73], and it seems logical that absolute increases in load carriage would generally have a greater impact on the biomechanics of women than men. This is not the case as it has been reported that men and women implement similar biomechanical adaptations to load carriage [21, 49]. It is possible the relatively small sample sizes in these studies may increase the chance of type II error, and sex differences may only occur with the development of greater muscle fatigue. A larger study with adequate statistical power is required to rigorously compare the effect of load carriage on the biomechanics between men and women and to identify if any differences are 
specifically related to sex, or a consequence of known sex differences in strength, stature and/or mass. A better understanding of sex differences in biomechanics during load carriage, and the effect of load carriage on the biomechanical changes induced by stride lengths and stride frequencies, is important when designing training protocols, developing strategies to mitigate injury risk, and optimising the performance of women in arduous roles.

\section{Conclusions}

This narrative review explores the influence of sex and stature on lower limb biomechanics, when walking at a fixed speed, stride length or stride frequency, and during load carriage. Marching 'in-step' at a fixed pace and stride length can result in over-striding in shorter individuals, increasing GRFs, muscle forces, and injury risk, particularly at speeds close to the preferred walk-to-run transition speed. Load carriage affects movement patterns and increases ground reaction forces, joint moments and the demands on the muscles. Many of these biomechanical responses are associated with injuries typically seen in military populations. It is unknown whether men and women adapt their biomechanics differently when speed, stride length and frequency are enforced, or whether these biomechanical adaptations are related to stature rather than sex.

\section{References}

1. Lloyd-Williams, R.C. and G. Fordy, The consequences of burden on the dismounted soldier. 2013, Defence Science and Technology Laboratory UK: Unpublished.

2. Knapik, J., E. Harman, and K. Reynolds, Load carriage using packs: A review of physiological, biomechanical and medical aspects. Applied Ergonomics, 1996. 27(3): p. 207-216.

3. Orr, R.M., et al., Soldier occupational load carriage: a narrative review of associated injuries. International Journal of Injury Control and Safety Promotion, 2014. 21(4): p. 388-396.

4. Knapik, J.J. and K. Reynolds, Load Carriage-Related Injury Mechanisms, Risk Factors, and Prevention, in The Mechanobiology and Mechanophysiology of Military-Related Injuries, A. Gefen and Y. Epstein, Editors. 2016, Springer International Publishing: Cham. p. 107-137.

5. Andersen, K.A., et al., Musculoskeletal Lower Limb Injury Risk in Army Populations. Sports Medicine - Open, 2016. 2(1): p. 22.

6. Blacker, S.D., et al., Risk factors for training injuries among British Army recruits. Military medicine, 2008. 173(3): p. 278-286.

7. Jones, B.H. and J.J. Knapik, Physical training and exercise-related injuries. Sports medicine, 1999. 27(2): p. 111-125.

8. Bergman, B.P. and S.A. St J Miller, Equal opportunities, equal risks? Overuse injuries in female military recruits. Journal of Public Health, 2001. 23(1): p. 35-39.

9. Ministry of Defence, Interim Report on the Health Risks to Women in Ground Close Combat Roles. 2016, Department of Manning (Army): Andover, UK.

10. Wilson, S. and D. Usher, TIN 3.182 Dismounted Anthropometric Data Collection. 2017, Defence Human Capability Science and Technology Centre: Porton Down, Salisbury Wilts, SP4 0JQ.

11. Ministry of Defence. UK armed forces biannual diversity statistics: 2019. 2019; Available from: https://www.gov.uk/government/statistics/uk-armed-forces-biannual-diversitystatistics-2019.

12. Seay, J.F., et al., Lower Extremity Mechanics During Marching at Three Different Cadences for 60 Minutes. Journal of Applied Biomechanics, 2014. 30(1): p. 21-30. 
13. Pires, N.J., B.S. Lay, and J. Rubenson, Joint-level mechanics of the walk-to-run transition in humans. Journal of Experimental Biology, 2014. 217(Pt 19): p. 3519-27.

14. Ardestani, M.M., et al., From normal to fast walking: Impact of cadence and stride length on lower extremity joint moments. Gait \& Posture, 2016. 46: p. 118-125.

15. Hreljac, A., Preferred and energetically optimal gait transition speeds in human locomotion. Medicine \& Science in Sports \& Exercise, 1993. 25(10): p. 1158-1162.

16. Weyand, P.G., et al., The mass-specific energy cost of human walking is set by stature. Journal of Experimental Biology, 2010. 213(23): p. 3972-3979.

17. Hreljac, A., Effects of physical characteristics on the gait transition speed during human locomotion. Human Movement Science, 1995. 14(2): p. 205-216.

18. Sentija, D., M. Rakovac, and V. Babic, Anthropometric characteristics and gait transition speed in human locomotion. Human Movement Science, 2012. 31(3): p. 672-682.

19. Boffey, D., et al., The Physiology and Biomechanics of Load Carriage Performance. Military Medicine, 2019: p. usy218-usy218.

20. Rigas, C., Spatial parameters of gait related to the position of the foot on the ground. Prosthetics and orthotics international, 1984. 8(3): p. 130-134.

21. Silder, A., S.L. Delp, and T. Besier, Men and women adopt similar walking mechanics and muscle activation patterns during load carriage. Journal of Biomechanics, 2013. 46(14): p. 2522-8.

22. Kung, S.M., et al., What factors determine the preferred gait transition speed in humans? A review of the triggering mechanisms. Human Movement Science, 2018. 57: p. 1-12.

23. Hill, P.F., et al., Stress Fracture of the Pubic Ramus in Female Recruits. The Journal of Bone and Joint Surgery. British volume, 1996. 78-B(3): p. 383-386.

24. Kelly, E.W., et al., Stress fractures of the pelvis in female navy recruits: an analysis of possible mechanisms of injury. Military Medicine, 2000. 165(2): p. 142-146.

25. $\mathrm{Xu}, \mathrm{C}$., et al., A cross-sectional study of the effects of load carriage on running characteristics and tibial mechanical stress: implications for stress-fracture injuries in women. BMC musculoskeletal disorders, 2017. 18(1): p. 125-125.

26. Hsiang, S.M. and C. Chang, The effect of gait speed and load carrying on the reliability of ground reaction forces. Safety Science, 2002. 40(7): p. 639-657.

27. Sun, D., et al., The effect of walking speed on the foot inter-segment kinematics, ground reaction forces and lower limb joint moments. PeerJ, 2018. 2018(8).

28. Birrell, S. and R.H. Hooper, The Biomechanics of Military Load Carriage and Injury Potential. 2005, Loughborough University.

29. Zadpoor, A.A. and A.A. Nikooyan, The relationship between lower-extremity stress fractures and the ground reaction force: A systematic review. Clinical Biomechanics, 2011. 26(1): p. 2328.

30. Martin, P.E. and A.P. Marsh, Step length and frequency effects on ground reaction forces during walking. Journal of Biomechanics, 1992. 25(10): p. 1237-1239.

31. Lim, Y.P., Y.C. Lin, and M.G. Pandy, Effects of step length and step frequency on lower-limb muscle function in human gait. Journal of Biomechanics, 2017. 57: p. 1-7.

32. Anderson, F.C. and M.G. Pandy, Individual muscle contributions to support in normal walking. Gait \& Posture, 2003. 17(2): p. 159-169.

33. Browning, R.C., et al., Effects of obesity and sex on the energetic cost and preferred speed of walking. Journal of Applied Physiology, 2006. 100(2): p. 390-398.

34. Harman, E., et al., The effects of walking speed on the biomechanics of backpack load carriage. 2000, US Army Research Institute of Environmental Medicine.

35. Hreljac, A., et al., When does a gait transition occur during human locomotion? Journal of sports science \& medicine, 2007. 6(1): p. 36-43.

36. Raynor, A.J., et al., Are transitions in human gait determined by mechanical, kinetic or energetic factors? Human Movement Science, 2002. 21(5): p. 785-805. 
37. Hreljac, A., Determinants of the gait transition speed during human locomotion: kinetic factors. Gait \& Posture, 1993. 1(4): p. 217-223.

38. Ranisavljev, I., et al., The relationship between allometry and preferred transition speed in human locomotion. Human Movement Science, 2014. 34: p. 196-204.

39. Segers, V., et al., Spatiotemporal characteristics of the walk-to-run and run-to-walk transition when gradually changing speed. Gait \& Posture, 2006. 24(2): p. 247-254.

40. Abe, D., Y. Fukuoka, and M. Horiuchi, Why do we transition from walking to running? Energy cost and lower leg muscle activity before and after gait transition under body weight support. PeerJ, 2019. 7: p. e8290.

41. Prilutsky, B.I. and R.J. Gregor, Swing-and support-related muscle actions differentially trigger human walk-run and run-walk transitions. Journal of Experimental Biology, 2001. 204(13):

p. 2277-2287.

42. $\quad$ Ministry of Defence, Military Annual Training Test (MATT) 2, in MATT-2. 2019.

43. Pope, R.P., Prevention of pelvic stress fractures in female army recruits. Military medicine, 1999. 164(5): p. 370.

44. Birrell, S.A. and R.A. Haslam, The effect of military load carriage on 3-D lower limb kinematics and spatiotemporal parameters. Ergonomics, 2009. 52(10): p. 1298-1304.

45. Dames, K.D. and J.D. Smith, Effects of load carriage and footwear on spatiotemporal parameters, kinematics, and metabolic cost of walking. Gait \& Posture, 2015. 42(2): p. 122-6.

46. Dames, K.D. and J.D. Smith, Effects of load carriage and footwear on lower extremity kinetics and kinematics during overground walking. Gait \& Posture, 2016. 50: p. 207-211.

47. Demura, T. and S. Demura, Relationship among gait parameters while walking with varying loads. Journal of Physiological Anthropology, 2010. 29(1): p. 29-34.

48. Huang, T.W. and A.D. Kuo, Mechanics and energetics of load carriage during human walking. Journal of Experimental Biology, 2014. 217(Pt 4): p. 605-13.

49. Krupenevich, R., et al., Males and Females Respond Similarly to Walking With a Standardized, Heavy Load. Military Medicine, 2015. 180(9): p. 994-1000.

50. LaFiandra, M., et al., How do load carriage and walking speed influence trunk coordination and stride parameters? Journal of Biomechanics, 2003. 36(1): p. 87-95.

51. Ling, W., et al., Women's load carriage performance using modular lightweight load-carrying equipment. Military medicine, 2004. 169(11): p. 914-919.

52. Attwells, R.L., et al., Influence of carrying heavy loads on soldiers' posture, movements and gait. Ergonomics, 2006. 49(14): p. 1527-37.

53. Majumdar, D., M.S. Pal, and D. Majumdar, Effects of military load carriage on kinematics of gait. Ergonomics, 2010. 53(6): p. 782-91.

54. Martin, P.E. and R.C. Nelson, Effects of Gender, Load, and Backpack on the Temporal and Kinematic Characteristics of Walking Gait. 1982, US Army Natick Research and Development Laboratories.

55. Armstrong, N.C., et al., The effects of body armour and load carriage of varying masses on respiratory function, movement and biomechanics and performance during a simulated military task in male and female soldiers. 2017, Defence Science and Technology Laboratory: Porton Down, Salisbury, Wiltshire SP4 0JQ.

56. Martin, P.E. and R.C. Nelson, The effect of carried loads on the walking patterns of men and women. Ergonomics, 1986. 29(10): p. 1191-202.

57. Liew, B., S. Morris, and K. Netto, The Effect of Backpack Carriage on the Biomechanics of Walking: A Systematic Review and Preliminary Meta-Analysis. Journal of Applied Biomechanics, 2016. 32(6): p. 614-629.

58. Harman, E., et al., The effects of backpack weight on the biomechanics of load carraige. 2000, US Army Research Institute of Environmental Medicine. 
59. Polcyn, A.F., et al., The effects of load weight: a summary analysis of maximal performance, physiological, and biomechanical results from four studies of load-carriage systems. 2001, Army Natick Soldier Center MA Supporting Sceince and Technolody Directorate.

60. Polcyn, A.F., et al., Effects of Weight Carried by Soldiers: Combined Analysis of Four Studies on Maximal Performance, Physiology and Biomechanics. 2002, US Army Research Institute of Environmental Medicine.

61. Wang, H., et al., The effects of load carriage and muscle fatigue on lower-extremity joint mechanics. Research Quarterly for Exercise and Sport, 2013. 84(3): p. 305-12.

62. Yang, X., et al., Biomechanics analysis of human walking with load carriage. Technology and Health Care, 2015. 23 Suppl 2: p. S567-75.

63. Neely, F.G., Intrinsic Risk Factors for Exercise-Related Lower Limb Injuries. Sports Medicine, 1998. 26(4): p. 253-263.

64. Blacker, S.D., D.M. Wilkinson, and M.P. Rayson, Gender differences in the physical demands of British Army recruit training. Military medicine, 2009. 174(8): p. 811-816.

65. Fallowfield, J.L., et al., Risk of Injury in Royal Air Force Training: Does Sex Really Matter? Military Medicine, 2018: p. usy177-usy177.

66. Seay, J.F., Biomechanics of Load Carriage--Historical Perspectives and Recent Insights. Journal of Strength \& Conditioning Research, 2015. 29 Suppl 11: p. S129-33.

67. O'Leary, T.J., et al., Sex differences in training loads during british army basic training. Medicine and Science in Sports and Exercise, 2018. 50(12): p. 2565-2574.

68. Wang, H., et al., Influence of Fatigue and Load Carriage on Mechanical Loading During Walking. Military Medicine, 2012. 177(2): p. 152-156.

69. Birrell, S.A., R.H. Hooper, and R.A. Haslam, The effect of military load carriage on ground reaction forces. Gait \& Posture, 2007. 26(4): p. 611-4.

70. Quesada, P.M., et al., Biomechanical and metabolic effects of varying backpack loading on simulated marching. Ergonomics, 2000. 43(3): p. 293-309.

71. Lenton, G.K., et al., Primarily hip-borne load carriage does not alter biomechanical risk factors for overuse injuries in soldiers. Journal of Science and Medicine in Sport, 2019. 22(2): p. 158-163.

72. Castro, M.P., et al., The influence of gait cadence on the ground reaction forces and plantar pressures during load carriage of young adults. Applied Ergonomics, 2015. 49: p. 41-46.

73. O'Leary, T.J., et al., Sex differences in neuromuscular fatigability in response to load carriage in the field in British Army recruits. Journal of Science and Medicine in Sport, 2018. 21(6): p. 591-595. 